\title{
A Compact Waveguide Slot Filtering Antenna Based on Mushroom-Type Surface
}

\author{
Zhi Zheng, Xiaochuan Fang, Wei Wang, Guanlong Huang, Senior Member, IEEE, Hongtao Zhang, \\ Xianling Liang, Senior Member, IEEE
}

\begin{abstract}
A mushroom-type surface based waveguide slot filtering antenna is proposed herein. First, using a mushroom-type surface replacing the conventional metal plane in the bottom of a rectangular waveguide, a novel filtering waveguide is achieved. This filtering waveguide functions as a bandstop filter when the surface performs as a perfect magnetic conductor. Then, a filtering waveguide slot antenna is obtained by cutting radiating slots on the top of the filtering waveguide. Compared with a traditional waveguide slot antenna, the proposed antenna has nearly the same radiation characteristic while realizes an additional filter selectivity with almost no extra volume occupying. A prototype is demonstrated in $\mathrm{Ku}$-band with operating in 12.25-12.75GHz and rejecting over $14.0-14.5 \mathrm{GHz}$, which can be a candidate for receiving antenna of satellite communication. The measured results agree well with the designed ones, showing a suppression level stronger than $40 \mathrm{~dB}$ in the rejecting band.
\end{abstract}

Index Terms-Filtering antenna, Mushroom-type surface, Waveguide slot antenna, Artificial material.

\section{INTRODUCTION}

$\mathrm{W}$ ITH the prosperous development of wireless communication systems, it is an irreversible trend for the microwave radio frequency (RF) front-end to become compact and multifunctional. As a result, a filtering antenna, integrating the antenna and filter in a single module and having both radiating and filtering functions simultaneously, has been proposed. The filtering antenna is able to effectively reduce the overall size and loss of the RF front-end, and it has attracted extensive research interests.

Many kinds of filtering antennas and their design methods have been reported in the literature [1]-[6]. By adding particular structures to antennas, such as parasitic loops [1] and shorting pins [2], some filtering antennas were realized. Inserting bandpass filter [3] or filtering power divider [4] to the feeding ports of their respective antennas is also an effective design method to conduct filtering antennas. Recently, a co-design approach with synthesizing an antenna as the last stage of a

This work was supported by the National Natural Science Foundation of China under Grant 61671416. (Corresponding author: Wei Wang.).

Z. Zheng, W. Wang, and H. Zhang are with the East China Research Institute of Electronic Engineering, Hefei, China (king-z-love@163.com, shu00ww@163.com, zhangfan0826@126.com).

$\mathrm{X}$. Fang is with the School of Electronic Engineering and Computer Science, Queen Mary University of London, London, UK. (x.fang@qmul.ac.uk).

G. Huang is with the College of Electronics and Information Engineering, Shenzhen University, Shenzhen, China (guanlong.huang@szu.edu.cn).

$X$. Liang is with the School of Communication and Information Engineering, Shanghai Jiao Tong University, Shanghai, China (liangxl@sjtu.edu.cn). multi-stage filter has been presented [5]-[6]. However, most of these filtering antennas were microstrip antennas based on single- or multi-layer dielectric-slabs, and the dielectric loss become larger for higher frequencies. Additionally, usually the filtering circuits or structures were unpackaged and exposed out in the previous design, which would introduces severe mutual coupling problem among adjacent elements for an array design.

Waveguide slot antenna has been widely used due to its advantages of high efficiency, especially at sub-millimeter or millimeter wave band, low profile, high mechanical strength, and high power-handling capability. There were a few researches about waveguide slot filtering antennas. To realize a filtering response, the waveguide-type filter was integrated into the waveguide cavity natively provided by the power divider feeding waveguide in [7] and [8]. While in [9], a single rectangular grating waveguide with metallic sheets was designed both as radiating cavity and filter. In our previous work [10], bed of metal nails has been embedded into a rectangular waveguide to achieve a bandstop filtering response. However, the height of the nails is about a quarter of the wavelength of the central frequency in the stopband, which is still too thick for some applications, and the structure of bed of nails is still quite complicated for fabrication, so it is necessary to design a more compact and simple structure further.

Mushroom-type surface was first proposed in 1999 [11] and has been widely used to design high-performance antennas [12]. In this study, we use the mushroom-type surface to design a waveguide slot filtering antenna. Compared with our previous work in [10], the proposed antenna here has lower profile, more design freedom, and easier fabrication. This is because that the mushroom-type surface can be achieved based on a thin dielectric substrate and its complex shaped metallization can be easily realized using printed-circuit-board (PCB) technology. The proposed filtering antenna is designed for $\mathrm{Ku}$-band, which operates in $12.25-12.75 \mathrm{GHz}$, while possesses the filtering ability to suppress the interference single from $14.0-14.5 \mathrm{GHz}$.

\section{Antenna Configuration, Mechanism, And Design}

\section{A. Antenna configuration}

The structure of the proposed filtering antenna is depicted in Fig. 1. A mushroom-type surface is placed on the waveguide cavity bottom of a common six-slot waveguide slot antenna. The surface is implemented on a RO4003C substrate with permittivity of 3.55 and thickness of $0.813 \mathrm{~mm}$. Printed square patches and via-holes for shorting the center of the patch to the 


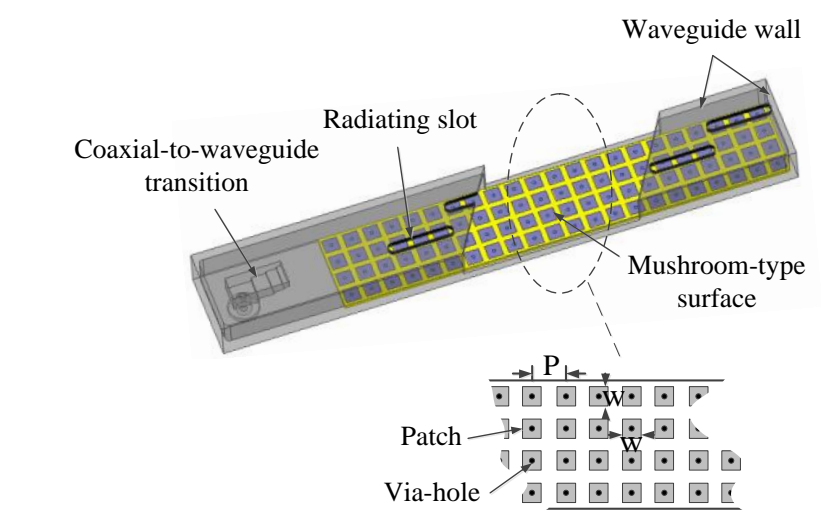

(a)

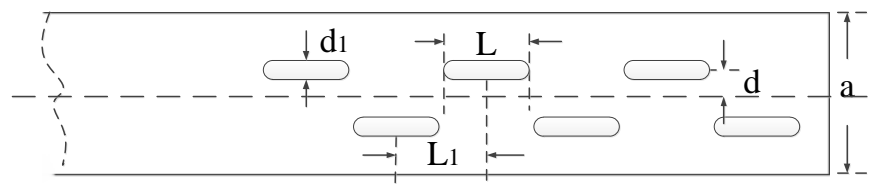

(b)

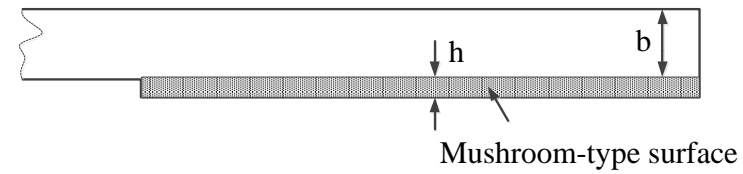

(c)

Fig. 1. Configuration of proposed filtering antenna. (a) 3-D perspective view. (b) Top view. (c) Side view.

TABLE I

DIMENSIONS OF THE FILTERING ANTENNA (UNIT: mm)

\begin{tabular}{cccccccc}
\hline \hline$a$ & $b$ & $d$ & $d_{1}$ & $L$ & $L_{1}$ & $P$ & $W$ \\
\hline 16.0 & 4.5 & 2.6 & 2.0 & 12.8 & 12.9 & 4.0 & 2.9 \\
\hline \hline
\end{tabular}

bottom metallic plane are periodic distributed. And a coaxial-to-waveguide transition is used to excite the filtering antenna. The major structural dimensions of the antenna are symbolized in Fig. 1, numerically determined, and listed in Table I.

\section{B. Filtering mechanism}

As well known, parallel-plate transmission has a cutoff frequency when one of the two perfect electric conductor (PEC) plates is substituted by a perfect magnetic conductor (PMC). The cutoff performance arrives when the distance between the two parallel plates is shorter than a quarter of the wavelength [13].

Inspired from this cutoff property, it is feasible to achieve a filtering waveguide structure by replacing the bottom stable PEC surface of a rectangular waveguide with a PEC/PMC changeable specially-designed surface, i.e., the mentioned mushroom-type surface. With the change of frequency, the mushroom-type surface transforms its EM function from PEC to PMC. Following this transforming, the filtering waveguide realizes passband and stopband simultaneously for different frequencies. Then a filtering waveguide slot antenna will be conducted by cutting radiating slots on the top of the proposed filtering waveguide. (a)

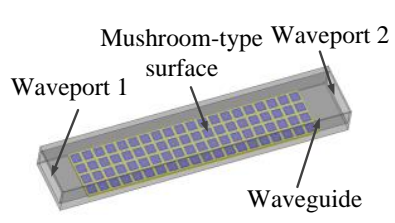

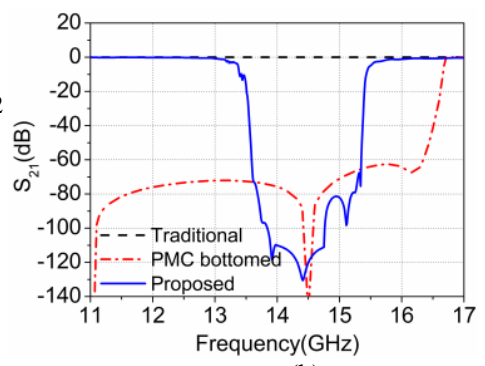

(b)
Fig. 2. (a) Configuration of proposed filtering waveguide and (b) its transmission coefficient compared with a traditional rectangular waveguide and a PMC bottomed waveguide (These three waveguides have the same cross-section dimensions $a$ and $b$ ).

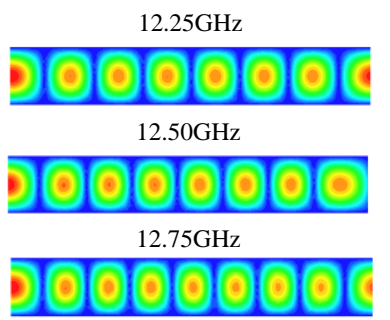

(a)

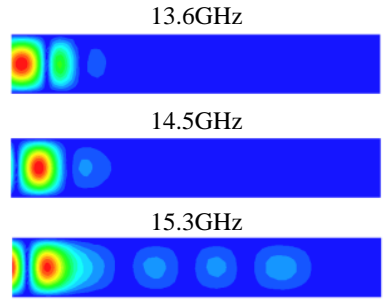

(b)
Fig. 3. Field distribution of the filtering waveguide: (a) in operating band; (b) in stopband.

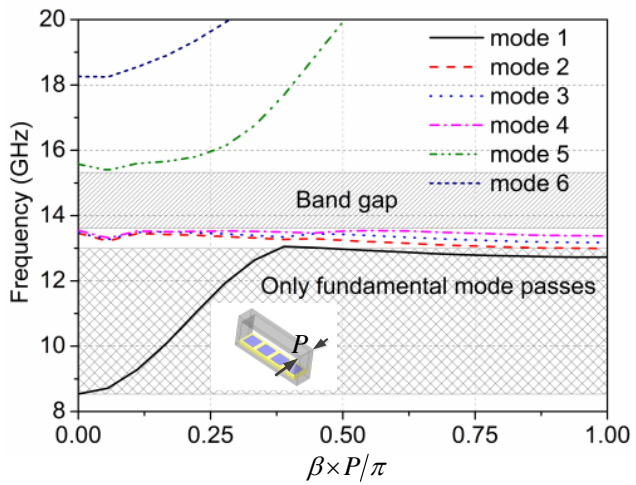

Fig. 4. Dispersion diagram of the $1 \mathrm{D}$-periodic structure in the filtering waveguide.

\section{Design of filtering waveguide}

The filtering waveguide is designed with a section of the proposed mushroom-type surface replacing parts of the bottom metal plane of a rectangular waveguide, as shown in Fig. 2(a).

Fig. 2(b) demonstrates the transmission coefficients $\left(S_{21}\right)$ of the proposed filtering waveguide as well as the comparison with the waveguides of pure PEC and PMC bottoms. For the frequencies before $13.0 \mathrm{GHz}$ and after $16.0 \mathrm{GHz}$, the $S_{21}$ curve of the filtering waveguide overlaps with that of traditional PEC waveguide. The nearly $0 \mathrm{~dB}$ performance implies that the wave propagates freely, as illuminated in Fig. 3(a), achieving a passband. While in Fig. 2(b), the stronger than $60 \mathrm{~dB}$ attenuation during $13.6-15.3 \mathrm{GHz}$ brings a stopband for the proposed filtering waveguide, in which the wave deteriorates sharply, as shown in Fig. 3(b). In the stopband, the proposed filtering waveguide works like a PMC bottomed waveguide. 
Overall, the filtering waveguide integrates the functions of a traditional PEC waveguide and a PMC bottomed waveguide depending on the PEC/PMC behaves of the mushroom-type surface.

Moreover, Fig. 4 calculates the dispersion curves of the first six modes for the mushroom-type surface based waveguide. As seen in Fig. 4, only mode 1, i.e., the fundamental mode, exists during 8.5-13.0GHz. Any frequencies during this band could be selected as the operating frequencies for the further filtering antenna design. In our work, the operating band is designed to be $12.25-12.75 \mathrm{GHz}$. The field distribution of the filtering waveguide during this operating band has been shown in Fig. 3(a). Clearly, this distribution is very similar to the $\mathrm{TE}_{10}$ mode of a common metal rectangular waveguide. Additionally, the entire loss of the filtering waveguide, including reflection loss and insertion loss, is less than $0.5 \mathrm{~dB}$ for the operating band of 12.25-12.75GHz, as indicated in Fig. 2(b).

Also as observed in Fig. 4, a band gap arises between mode 4 and mode 5 , from $13.6 \mathrm{GHz}$ to $15.3 \mathrm{GHz}$, in which the wave propagation will be blocked by the filtering waveguide. Absolutely, the gap coincides with the high attenuation area of $\mathrm{S}_{21}$ performance shown in Fig. 2(b). And the pre-designed $14.0-14.5 \mathrm{GHz}$ rejecting frequencies of the filtering antenna is included in the band gap.

Besides, in our presented design, the location of the stopband can be easily adjusted to meet different demands. Fig. 5 shows the effect of the parameter $W$ of the mushroom-type surface (as labeled in Fig. 1(a)) on the stopband. It is observed that, as $W$ is decreased, the stopband moves to higher frequencies.

\section{Design of filtering antenna}

By carefully designing the offset and length of longitudinal slots on top of the proposed filtering waveguide, a waveguide slot filtering antenna is obtained, and its configuration has been shown in Fig. 1. The operating frequencies of the proposed antenna are centered at $12.5 \mathrm{GHz}$, over $12.25-12.75 \mathrm{GHz}$, and its stopband is designed to cover $14.0-14.5 \mathrm{GHz}$. Since only the $\mathrm{TE}_{10}$ like fundamental mode works for the operating band as mentioned above, the design method of radiating slot is the same as that of a traditional waveguide slot antenna [14].

Fig. 6 depicts the simulated reflection coefficient of the proposed antenna. Within the operating band from $12.25 \mathrm{GHz}$ to $12.75 \mathrm{GHz}$, good impedance matching with $\left|S_{11}\right|<-12.5 \mathrm{~dB}$ is obtained. While $S_{11}$ performs nearly $0 \mathrm{~dB}$ for the stopband of $14.0-14.5 \mathrm{GHz}$, in which the input signals are almost totally reflected or called rejected.

To quantify the filtering ability of the proposed antenna further, a traditional waveguide slot antenna is used as the reference. The referential antenna, as shown in Fig. 7, is well-designed to work in the same operating band as the proposed one and also to have six radiating slots.

Fig. 8 exhibits the simulated gain responses verse frequency for both the proposed antenna and the traditional one. As can be observed, for the proposed antenna, its broadside gain is flat about $13.6 \mathrm{dBi}$ in the operating band while varies from $-45 \mathrm{dBi}$

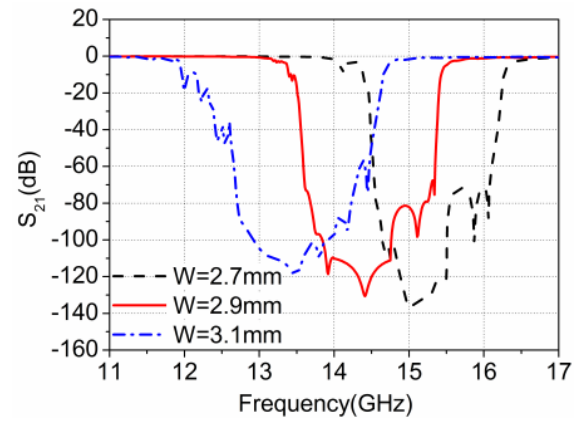

Fig. 5. Stopband characteristics of the filtering waveguide with different printed patch width $W$.

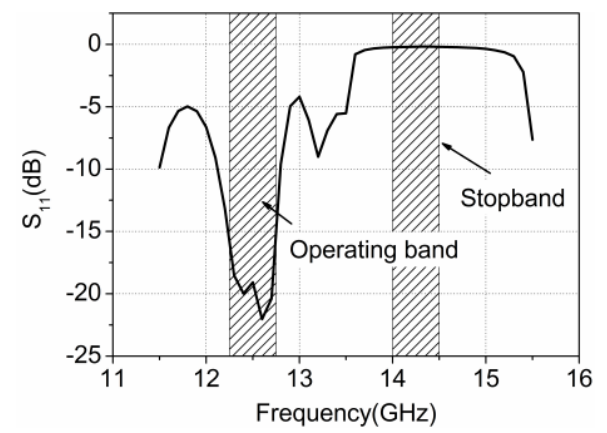

Fig. 6. Simulated reflection coefficient of proposed filtering antenna.

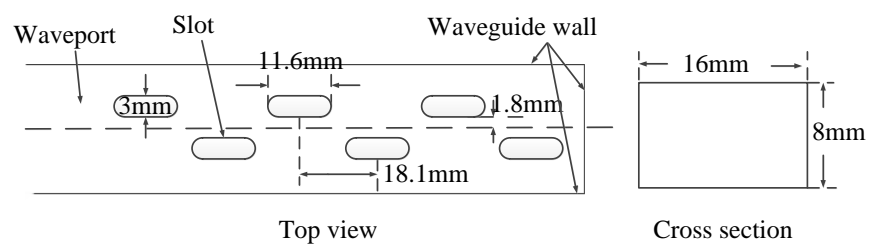

Fig. 7. Configuration of referential waveguide slot antenna.

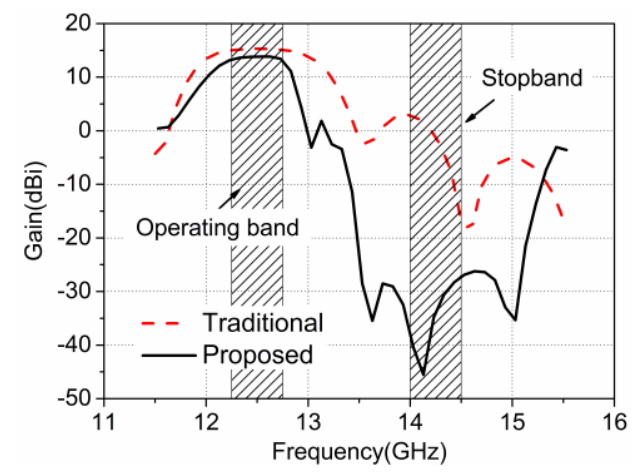

Fig. 8. Comparison of broadside gain response versus frequency between the proposed antenna and the traditional antenna.

to $-26.5 \mathrm{dBi}$ during the rejecting band. Comparing the gain values in these two bands, a suppression level more than $40 \mathrm{~dB}$ is obtained for our proposed antenna.

Also as shown in Fig. 8, the in-band broadside gain of the traditional antenna is around $15.1 \mathrm{dBi}$. It is $1.5 \mathrm{~dB}$ higher than that of the proposed antenna mainly due to the larger antenna aperture. The maximum value of the gain in the rejecting band is $2.9 \mathrm{dBi}$ for the traditional antenna, which arises at $14.0 \mathrm{GHz}$. Compared with the corresponding value of $-26.5 \mathrm{dBi}$ for the 


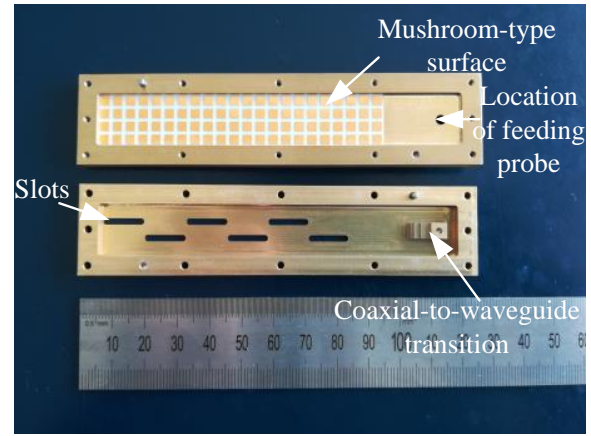

Fig. 9 Inner view of the fabricated waveguide slot filtering antenna.

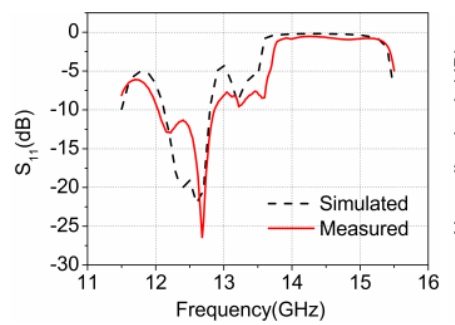

(a)

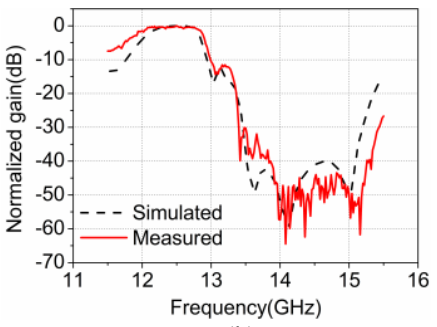

(b)
Fig. 10. Simulated and measured results of the filtering antenna: (a) reflection coefficients; (b) normalized gain.

proposed antenna, a nearly $30 \mathrm{~dB}$ improvement of out-of-band rejection is obtained for our filtering antenna.

\section{MEASURED Results}

For demonstration, the $k u$-band prototype antenna has been fabricated and measured. The top metal cover (with etching slots) and the bottom cavity (with embedded mushroom-type surface) are first milled separately, as shown in Fig. 9, and then fixed together with screws surrounding the edges.

Fig. 10(a) describes the simulated and measured reflection coefficient of the proposed antenna. As observed clearly, the measured result agrees well with the simulated one, obtaining an $\left|S_{11}\right|<-10 d B$ operating band from $12.1 \mathrm{GHz}$ to $12.8 \mathrm{GHz}$. And a predicted nearly 0 -dB $S$-parameter performance $\left(\left|S_{11}\right|>-1.0 d B\right)$ realizes a stopband from $13.9 \mathrm{GHz}$ to $15.2 \mathrm{GHz}$.

Both the measured operating and rejecting bands cover the pre-specified bands. The small discrepancy is mainly due to the fabrication tolerances.

Fig. 10(b) plots the simulated and measured broadside gain responses of the proposed antenna. They agree well with each other. The measured normalized gain in the rejecting band is below $-40 \mathrm{~dB}$, achieving a suppression more than $40 \mathrm{~dB}$.

Fig. 11 shows the simulated and measured $H$-plane radiation patterns of the proposed antenna at $12.25 \mathrm{GHz}, 12.50 \mathrm{GHz}$ and $12.75 \mathrm{GHz}$. The measured radiation patterns show a good agreement with the simulated ones. They are classical patterns of a one-dimensional six-element linear array, and in turn verify the good radiation properties of the designed filtering antenna.

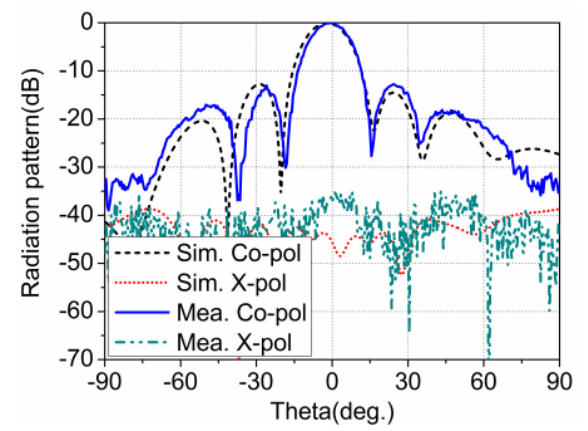

(a)

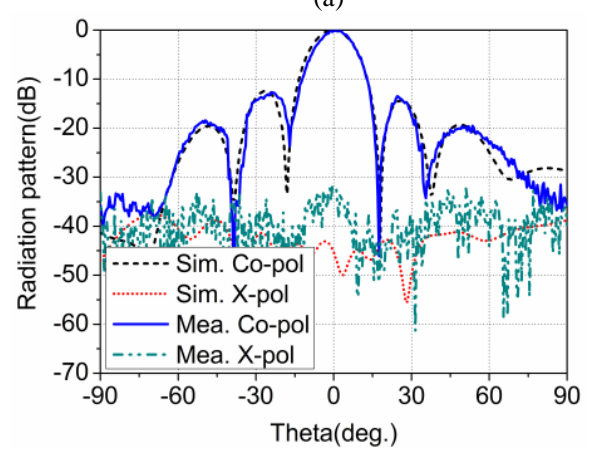

(b)

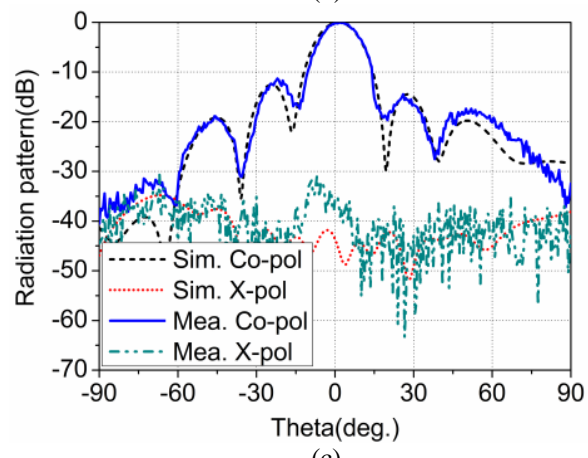

(c)

Fig. 11. Simulated and measured $\mathrm{H}$-plane radiation patterns of the filtering antenna: (a) $12.25 \mathrm{GHz}$; (b) $12.5 \mathrm{GHz}$; (c) $12.75 \mathrm{GHz}$.

\section{CONCLUSION}

A novel waveguide slot filtering antenna based on mushroom-type surface has been presented in this study. By placing the mushroom-type surface in the cavity bottom of a traditional waveguide slot antenna, an additional stopband filtering function is achieved. Since the mushroom-type surface is achieved on a thin dielectric substrate, almost no addition space is occupied, and then the filtering waveguide slot antenna owns almost the same low profile property as a traditional waveguide slot antenna. A $k u$-band prototype was fabricated and measured for demonstration. The measured results show a suppressing ability more than $40 \mathrm{~dB}$ in the rejecting band. Moreover, because of the mature PCB technology, more complex metallization can be easily fabricated on the dielectric substrate, to achieve better radiation and filtering performances further. 


\section{REFERENCES}

[1] J. Wu, Z. Zhao, Z. Nie, and Q. Liu, "A printed unidirectional antenna with improved upper band-edge selectivity using a parasitic loop," IEEE Trans. Antennas Propag., vol. 63, no. 4, pp. 1832-1837, Apr. 2015.

[2] S. Wong, T. Huang, C. Mao, Z. Chen, and Q. Chu, "Planar filtering ultra-wideband (UWB) antenna with shorting pins," IEEE Trans. Antennas Propag., vol. 61, no. 2, pp. 948-953, Feb. 2013.

[3] C. Wu, C. Wang, S. Chen, and C. Chen, "Balanced-to-unbalanced bandpass filters and the antenna applications," IEEE Trans. Microw. Theory Tech., vol. 56, no. 11, pp. 2474-2482, Nov. 2008.

[4] F. Chen, H. Hu, R. Li, Q. Chu and M. J. Lancaster, "Design of filtering microstrip antenna array with reduced sidelobe level," IEEE, Trans. Antennas Propag., vol. 65, no. 2, pp. 903-908, 2017.

[5] C. K. Lin and S. J. Chung, "A compact filtering microstrip antenna with quasi-elliptic broadside antenna gain response," Antenna Wireless Propag. Lett., vol. 10, pp. 381-384, 2011.

[6] O. A. Nova, J. C. Bohorquez, N. M. Pena, G. E. Bridges, L. Shafai, and C. Shafai, "Filter-antenna module using substrate integrated waveguide cavities," Antennas Wireless Propag. Lett., vol. 10, pp. 59-62, 2011.

[7] X. Xu, M. Zhang, J. Hirokawa, and M.ando, "E-band plate-laminated waveguide filters and their integration into a corporate-feed slot array antenna with diffusion bonding technology," IEEE Trans. Microw. Theory Techn., vol. 64, no. 11, pp. 3592-3603, 2016.

[8] J. Lu, H. Zhang, W. Wang, X. Liang, J. Ge, M. Jin and W. Wu, "Broadband dual-polarized waveguide slot filtenna array with low cross polarization and high efficiency," IEEE, Trans. Antennas Propag., vol. 67, no. 1, pp. 151-159, 2019.

[9] W. Yuan, X. Liang, L. Zhang, J. Ge, W. Zhu, R. Jin, "Rectangular grating waveguide slot array antenna for SATCOM applications," IEEE Trans. Antennas Propagat., vol. 67, no. 6, pp. 3869-3880, 2019.

[10] W. Wang, Z. Zheng, X. Fang, H. Zhang, M. Jin, J. Lu, Q. Luo, and S. Gao, "A waveguide slot filtering antenna with an embedded metamaterial structure," IEEE Trans. Antennas Propagat., vol. 67, no. 5, pp. 2953-2960, 2019.

[11] D. Sievenpiper, L. Zhang, R. F. J. Broas, N. Alexopolous, and E. Yablonovitch, "High-impedance electromagnetic surfaces with a forbidden frequency band," IEEE Trans. Microw. Theory Techn., vol. 47, no. 11, pp. 2059-2074, 1999.

[12] A. Foroozesh and L. Shafai, "Investigation Into the Application of Artificial Magnetic Conductors to Bandwidth Broadening, Gain Enhancement and Beam Shaping of Low Profile and Conventional Monopole Antennas, "IEEE Trans. Antennas Propag., vol. 59, no. 1, pp. 4-20, 2011.

[13] P.-S. Kildal, E. Alfonso, A. Valero-Nogueira, and E. Rajo-Iglesias, "Local metamaterial-based waveguides in gaps between parallel metal plates," IEEE Antennas Wireless Propag. Lett., vol. 8, pp. 84-87, 2009.

[14] R. Elliott and L. Kurtz, "The design of small slot arrays," IEEE Trans. Antennas Propag., vol. 26, no. 2, pp. 214-219, Mar. 1987. 